\title{
Association of Antihypertensive Agents with the Risk of In-Hospital Death in Patients with Covid-19
}

\author{
Laurent Chouchana ${ }^{1}$ (1) - Nathanaël Beeker ${ }^{2}$. Nicolas Garcelon ${ }^{3,4} \cdot$ Bastien Rance $^{3,5} \cdot$ Nicolas Paris $^{6}$ - Elisa Salamanca ${ }^{6}$. \\ Elisabeth Polard ${ }^{7}$. Anita Burgun ${ }^{3,5,8}$. Jean-Marc Treluyer ${ }^{1,2}$. Antoine Neuraz ${ }^{3,8}$ - on behalf of AP-HP/Universities/ \\ Inserm COVID-19 research collaboration, AP-HP Covid CDR Initiative, and "Entrepôt de Données de Santé" AP-HP \\ Consortium"
}

Accepted: 8 February 2021 / Published online: 17 February 2021

(C) Springer Science+Business Media, LLC, part of Springer Nature 2021

\begin{abstract}
Purpose The role of angiotensin receptor blockers (ARB), angiotensin-converting enzyme inhibitors (ACEi), or other antihypertensive agents in the case of Covid-19 remains controversial. We aimed to investigate the association between antihypertensive agent exposure and in-hospital mortality in patients with Covid-19.

Methods We performed a retrospective multicenter cohort study on patients hospitalized between February 1 and May 15, 2020. All patients had been followed up for at least 30 days.

Results Of the 8078 hospitalized patients for Covid-19, $3686(45.6 \%)$ had hypertension and were included in the study. In this population, the median age was 75.4 (IQR, 21.5) years and $57.1 \%$ were male. Overall in-hospital 30 -day mortality was $23.1 \%$. The main antihypertensive pharmacological classes used were calcium channel blockers (CCB) $(n=1624,44.1 \%)$, beta-blockers $(n=1389,37.7 \%)$, ARB $(n=1154,31.3 \%)$, and ACEi $(n=998,27.1 \%)$. The risk of mortality was lower in CCB (aOR, $0.83[0.70-$ $0.99]$ ) and beta-blockers (aOR, 0.80 [0.67-0.95]) users and non-significant in ARB (aOR, 0.88 [0.72-1.06]) and ACEi (aOR, 0.83 [0.68-1.02]) users, compared to non-users. These results remain consistent for patients receiving CCB, beta-blocker, or ARB as monotherapies.

Conclusion This large multicenter retrospective of Covid-19 patients with hypertension found a reduced mortality among CCB and beta-blockers users, suggesting a putative protective effect. Our findings did not show any association between the use of renin-angiotensin-aldosterone system inhibitors and the risk of in-hospital death. Although they need to be confirmed in further studies, these results support the continuation of antihypertensive agents in patients with Covid-19, in line with the current guidelines.
\end{abstract}

Keywords Covid-19 · SARS-CoV-2 $\cdot$ Hypertension $\cdot$ Renin-angiotensin-aldosterone inhibitors $\cdot$ Drug safety $\cdot$ Calcium channel blockers

Laurent Chouchana

laurent.chouchana@aphp.fr

1 Centre Régional de Pharmacovigilance, Département de Pharmacologie, Hôpital Cochin, AP-HP.Centre - Université de Paris, 27 rue du Faubourg Saint-Jacques, 75014 Paris, France

2 Unité de Recherche clinique, Hôpital Cochin, AP-HP.Centre Université de Paris, Paris, France

3 Centre de Recherche des Cordeliers, INSERM UMRS 1138 Team 22, Université de Paris, Paris, France
4 Institut Imagine, Université de Paris, Paris, France

5 Département d'informatique médicale, Hôpital Européen Georges Pompidou, AP-HP.Centre - Université de Paris, Paris, France

6 Département Web Innovation Données (WIND), Direction des systèmes d'information, AP-HP, Paris, France

7 Centre Régional de Pharmacovigilance, pharmacoépidémiologie et information sur le médicament, CHU Rennes, Rennes, France

8 Département d'informatique médicale, Hôpital Necker-Enfants Malades, AP-HP.Centre - Université de Paris, Paris, France 


\section{Introduction}

Coronavirus disease-2019 (Covid-19), due to the novel severe acute respiratory syndrome coronavirus-2 (SARS-CoV-2), is a global pandemic representing a major threat to global health. Data from several cohorts have shown that age and cardiovascular comorbidities, including hypertension, were among the main determinants of severe disease [1,2]. As SARS-CoV-2 uses the angiotensin-converting enzyme 2 (ACE2) receptor as an entrance door into cells, questions have been raised about the role of the renin-angiotensin-aldosterone system (RAAS) activity on Covid-19 pathophysiology [3]. RAAS inhibitors directly act on RAAS in two different ways, both leading to ACE2 upregulation [3]. On the contrary, some authors have hypothesized that the use of RAAS inhibitors may protect against acute lung injury caused by angiotensin II accumulation [4]. Therefore, since these drugs such as ACE inhibitors (ACEi) or angiotensin receptor blockers (ARB) are common treatments for hypertension, controversies about their role on Covid-19 severity have been raised. To date, scientific societies have advised against their discontinuation in patients with hypertension, until robust clinical evidence is available [5]. Previous reports showed that RAAS inhibitors do not increase the risk of severe or fatal outcomes in Covid-19 patients $[1,6$, 7]. The role of other antihypertensive agents is unclear. However, these analyses are retrospective, and additional data are needed to ascertain the beneficial or harmful effect of antihypertensive agents on Covid-19 mortality.

In order to provide additional information, we performed a multicenter retrospective cohort study in patients hospitalized for Covid-19 in greater Paris area, France.

\section{Methods}

\section{Study Population}

This retrospective cohort study was conducted using the Assistance Publique-Hopitaux de Paris (AP-HP) Health Data Warehouse ("Entrepôt de Données de Santé (EDS)," https://eds.aphp.fr/). This data warehouse contains electronic health records (EHRs) of all inpatients from the 39 greater Paris university hospitals. Adult patients having at least one SARS-CoV-2-positive PCR test and hospitalized between February 1 and May 15, 2020, have been included. Patients having a PCR test performed more than 14 days after hospitalization have been excluded considering they were very likely to have nosocomial infection. Patients have been followed at least 30 days after inclusion (date of the first positive PCR test). The Institutional Review Board of the AP-HP Health Data Warehouse approved this study on April 7, 2020, under the number CSE-20-18_COVID19.

\section{Comorbidities and Drug Exposure}

Hypertension and other comorbidities have been retrieved in EHRs using specific codes from the International Classification of Diseases $10^{\text {th }}$ version, combined with natural language processing (regular expressions) as previously described, within the six months previous to the hospital stay for Covid-19 (Suppl Table S1) [8]. Antihypertensive agent exposure has been identified in EHRs at admission of the hospital stay for Covid-19 and within the six previous months, using both specific Anatomical Therapeutic Chemical (ATC) codes and deep learning algorithm on clinical narratives as previously described [8]. Antihypertensive agents considered in this study were identified using the specific ATC codes related to ARB, ACEi, calcium channel blockers (CCB), beta-blockers, and centrally acting sympatholytics (Suppl Table S2). As described in Neuraz et al. [8], we extracted drug mentions and their attributes (dose, frequency, duration, route, condition) using a deep-learning pipeline based on fine-tuned multilingual embedding and bidirectional long-short term memory units combined with conditional random fields [8]. The drug mentions were then normalized to ATC using exact string matching.

\section{Statistical Analysis}

The primary outcome was all-cause 30-day in-hospital mortality. We assessed the association between antihypertensive agent class exposure and primary outcome using a multivariate logistic regression. Analyses were adjusted on age, sex, and main chronic diseases to take into account confounding factors known to be associated with Covid-19 mortality. We further performed a sensitivity analysis considering exposure to the most frequent antihypertensive regimens, including monotherapies.

Data are presented as median (interquartile range [IQR]) and numbers (\%). The results of the logistic regression model are presented as crude odds ratio (OR) or adjusted OR (aOR), and their $95 \%$ confidence interval $([95 \% \mathrm{CI}])$. All analyses were performed using the $\mathrm{R}$ statistical software.

\section{Results}

In Greater Paris University Hospitals, 8078 patients had been hospitalized for Covid-19 between February 1 and May 15, 2020, among which 1531 (19.0\%) died within 30 days. Among the hospitalized patients, 3686 (45.6\%) had hypertension and were included in the study. In this population, the median age was 75.4 (IQR, 21.5) years, and $57.1 \%$ were male (Table 1). In patients with hypertension, the main comorbidities were diabetes $(n=1730,46.9 \%)$, other cardiovascular diseases $(n=1438,39 \%)$, and chronic kidney disease $(n=1407$, 
Table 1 Patient characteristics

All patients $(N=8078)$

\begin{tabular}{|c|c|c|c|c|}
\hline & \multirow[t]{2}{*}{ All patients $(N=8078)$} & \multicolumn{3}{|c|}{ Patients with hypertension } \\
\hline & & All $(N=3686)$ & Non-survivors $(N=853)$ & Survivors $(N=2833)$ \\
\hline \multicolumn{5}{|l|}{ Patient characteristics } \\
\hline Age-years, median (IQR) & $68(25.9)$ & $75.4(21.5)$ & $82.1(16.3)$ & $72.9(22.2)$ \\
\hline \multicolumn{5}{|l|}{ Age - distribution } \\
\hline $18-44$ years & $1020(12.6 \%)$ & $123(3.3 \%)$ & $5(0.6 \%)$ & $118(4.2 \%)$ \\
\hline $45-64$ & $2544(31.5 \%)$ & $876(23.8 \%)$ & $83(9.7 \%)$ & $793(28 \%)$ \\
\hline $65-74$ & $1662(20.6 \%)$ & $813(22.1 \%)$ & $165(19.3 \%)$ & $648(22.9 \%)$ \\
\hline $75-84$ & $1457(18 \%)$ & $919(24.9 \%)$ & $264(30.9 \%)$ & $655(23.1 \%)$ \\
\hline$>85$ & $1395(17.3 \%)$ & $955(25.9 \%)$ & $336(39.4 \%)$ & $619(21.8 \%)$ \\
\hline \multicolumn{5}{|l|}{ Sex } \\
\hline Female & $3291(40.7 \%)$ & $1583(42.9 \%)$ & $330(38.7 \%)$ & $1253(44.2 \%)$ \\
\hline Male & $4787(59.3 \%)$ & $2103(57.1 \%)$ & $523(61.3 \%)$ & $1580(55.8 \%)$ \\
\hline \multicolumn{5}{|l|}{ Chronic diseases } \\
\hline Hypertension & $3686(45.6 \%)$ & $3686(100 \%)$ & $853(100 \%)$ & $2833(100 \%)$ \\
\hline Chronic kidney disease & $1644(20.4 \%)$ & $1407(38.2 \%)$ & $384(45 \%)$ & $1023(36.1 \%)$ \\
\hline Cerebrovascular disease & $1511(18.7 \%)$ & $1209(32.8 \%)$ & $309(36.2 \%)$ & $900(31.8 \%)$ \\
\hline Cardiovascular disease & $1762(21.8 \%)$ & $1438(39 \%)$ & $378(44.3 \%)$ & $1060(37.4 \%)$ \\
\hline Cardiac failure & $1099(13.6 \%)$ & $949(25.7 \%)$ & $261(30.6 \%)$ & $688(24.3 \%)$ \\
\hline Diabetes & $2220(27.5 \%)$ & $1730(46.9 \%)$ & $404(47.4 \%)$ & $1326(46.8 \%)$ \\
\hline Respiratory disease & $1427(17.7 \%)$ & $944(25.6 \%)$ & $162(19.0 \%)$ & $782(27.6 \%)$ \\
\hline Obesity & $1851(22.9 \%)$ & $1072(29.1 \%)$ & $239(28.0 \%)$ & $833(29.4 \%)$ \\
\hline Malignancies & $1803(22.3 \%)$ & $1286(34.9 \%)$ & $324(38.0 \%)$ & $962(34.0 \%)$ \\
\hline \multicolumn{5}{|l|}{ Antihypertensive drugs } \\
\hline \multicolumn{5}{|l|}{ Pharmacological classes* } \\
\hline CCB users & $2210(27.4 \%)$ & $1624(44.1 \%)$ & $340(39.9 \%)$ & $1284(45.3 \%)$ \\
\hline Beta-blocker users & $1920(23.8 \%)$ & $1389(37.7 \%)$ & $315(36.9 \%)$ & $1074(37.9 \%)$ \\
\hline RAAS inhibitor users & $2720(33.7 \%)$ & $2043(55.4 \%)$ & $441(51.7 \%)$ & $1602(56.5 \%)$ \\
\hline ARB & $1520(18.8 \%)$ & $1154(31.3 \%)$ & $250(29.3 \%)$ & $904(31.9 \%)$ \\
\hline ACEi & $1337(16.6 \%)$ & $998(27.1 \%)$ & $214(25.1 \%)$ & $784(27.7 \%)$ \\
\hline Centrally acting sympatholytic users & $259(3.2 \%)$ & $172(4.7 \%)$ & $35(4.1 \%)$ & $137(4.8 \%)$ \\
\hline No antihypertensive drug & $3982(49.3 \%)$ & $826(22.4 \%)$ & $229(26.8 \%)$ & $597(21.1 \%)$ \\
\hline \multicolumn{5}{|l|}{ Treatment schemes } \\
\hline CCB only & $524(6.5 \%)$ & $313(8.5 \%)$ & $67(7.9 \%)$ & $246(8.7 \%)$ \\
\hline Beta-blocker only & $528(6.5 \%)$ & $298(8.1 \%)$ & $72(8.4 \%)$ & $226(8 \%)$ \\
\hline ARB only & $447(5.5 \%)$ & $303(8.2 \%)$ & $69(8.1 \%)$ & $234(8.3 \%)$ \\
\hline ACEi only & $281(3.5 \%)$ & $196(5.3 \%)$ & $42(4.9 \%)$ & $154(5.4 \%)$ \\
\hline Centrally acting sympatholytic only & $48(0.6 \%)$ & $7(0.2 \%)$ & $0(0 \%)$ & $7(0.2 \%)$ \\
\hline $\mathrm{CCB}$ and beta-blocker & $230(2.8 \%)$ & $171(4.6 \%)$ & $37(4.3 \%)$ & $134(4.7 \%)$ \\
\hline $\mathrm{ARB}$ and $\mathrm{CCB}$ & $400(5 \%)$ & $302(8.2 \%)$ & $61(7.2 \%)$ & $241(8.5 \%)$ \\
\hline ARB and beta-blocker & $203(2.5 \%)$ & $166(4.5 \%)$ & $36(4.2 \%)$ & $130(4.6 \%)$ \\
\hline $\mathrm{ACEi}$ and $\mathrm{CCB}$ & $329(4.1 \%)$ & $242(6.6 \%)$ & $48(5.6 \%)$ & $194(6.8 \%)$ \\
\hline ACEi and beta-blocker & $298(3.7 \%)$ & $212(5.8 \%)$ & $49(5.7 \%)$ & $163(5.8 \%)$ \\
\hline Other schemes & $808(10 \%)$ & $650(17.6 \%)$ & $143(16.8 \%)$ & $507(17.9 \%)$ \\
\hline No antihypertensive drug & $3982(49.3 \%)$ & $826(22.4 \%)$ & $229(26.8 \%)$ & $597(21.1 \%)$ \\
\hline
\end{tabular}

Non-survivors are considered in relation to in-hospital 30-day mortality

$A R B$ angiotensin II receptor blockers, $A C E i$ angiotensin-converting enzyme inhibitors, $C C B$ calcium channel blockers

*These pharmacological groups of users are not exclusive and one patient can be exposed to more than one pharmacological class 
$38.2 \%$ ). Regarding antihypertensive agents, the main pharmacological classes used were RAAS inhibitors $(n=2043$, $55.4 \%)$, CCB $(n=1624,44.1 \%)$, beta-blockers $(n=1154$, $37.7 \%)$, and centrally acting sympatholytics $(n=172$, $4.7 \%$ ). More specifically, for RAAS inhibitors, ARB and ACEi were used in 1154 (31.3\%) and 998 (27.1\%) patients, respectively, and no patient received a renin inhibitor. The main antihypertensive therapeutic schemes were monotherapies with a CCB or an ARB agent in 313 $(8.5 \%)$ and $303(8.2 \%)$ patients, respectively, and a combination therapy of ARB and CCB in $302(8.2 \%)$ patients (Table 1). In 826 patients (22.4\%), no antihypertensive agent was identified.

In total, there were $853(23.1 \%)$ all-cause in-hospital 30-day deaths. Patients who died were older and were more likely to be men, compared to survivors. They also had chronic diseases more frequently, except for respiratory diseases and obesity. The risk of mortality was significantly lower in CCB (aOR, 0.83 [0.70-0.99]) and beta-blocker (aOR, 0.80 [0.67-0.95]) users and nonsignificant in ARB (aOR, 0.88 [0.72-1.06]) and ACEi (aOR, 0.83 [0.68-1.02]) users, compared to non-users (Fig. 1, Suppl. Table S3). When restricting analysis to monotherapeutic schemes of antihypertensive agents, the results remain consistent for $\mathrm{CCB}$ (aOR, 0.69 [0.5-0.95]), beta-blocker (aOR, 0.67 [0.48-0.93], and ARB (aOR, 0.8 [0.57-1.11]) (Suppl. Figure S1).

\section{Discussion}

This study, one of the largest multicenter retrospective to date on more than 3600 hospitalized Covid-19 patients with hypertension, provides two main findings. First, there is a significant protective effect of CCB or beta-blocker use on the risk of death in hypertensive patients with Covid-19. Second, based on more than 2000 patients exposed to a RAAS inhibitor, there is no association with the use of ACEi or ARB and the risk of in-hospital death.

Hypertension and cardiovascular comorbidities have been previously reported as risk factors for severe Covid-19 and fatal outcome $[1,9,10]$. The underlying pathogenic mechanisms of these comorbidities remain unclear and may involve the RAAS as being a double-edged sword. On one hand, RAAS inhibitors increase the expression of ACE2, which could promote virus entry [3]. On the other hand, RAAS inhibitors, especially ARB, could reverse deleterious effects of unopposed angiotensin II accumulation resulting from ACE2 downregulation associated with SARS-CoV-2 entry in cells $[4,11]$. Clinical evidence based on a large study including 12,594 tested patients for SARS-CoV-2 showed no increase of likelihood to have a positive test among RAAS users [12]. Furthermore, in line with our findings, a meta-analysis on four retrospective studies that include 921 ACEi or ARB users found no difference in the risk of death compared to nonusers (OR, 0.88 [0.68-1.14]) [13]. In the context of the global
Fig. 1 In-hospital 30-day mortality in Covid-19 patients with hypertension according to antihypertensive drug exposure. These groups of users are not exclusive, and one patient can be exposed to more than one pharmacological class. Analyses have been adjusted on age, sex, and main chronic diseases (i.e., hypertension, chronic kidney disease, cerebrovascular disease, cardiovascular disease, cardiac failure, diabetes, respiratory disease, obesity, and malignancies). ARB, angiotensin II receptor blockers; ACEi, angiotensin-converting enzyme inhibitors; $\mathrm{CCB}$, calcium channel blockers

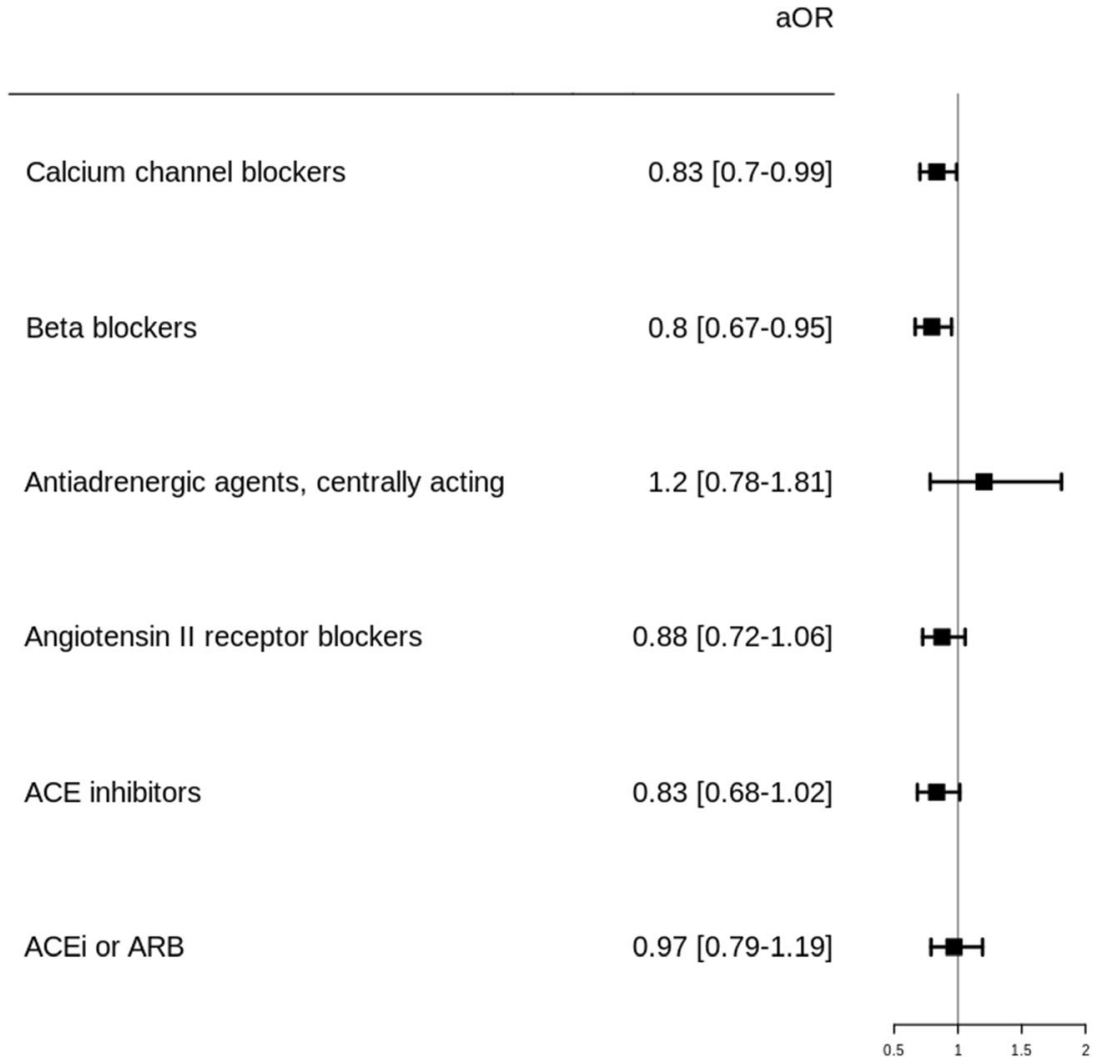


pandemic of Covid-19, these findings are reassuring as RAAS inhibitors are major treatments of hypertension used in millions of patients around the world.

In our study, we found that $\mathrm{CCB}$ and beta-blockers were more frequently used in survivors (OR 0.83 [0.70-0.99] and 0.80 [0.67-0.95], respectively) than in non-survivors. These findings were statistically significant. Previous Covid-19 cohort studies did not found any beneficial or deleterious effect of CCB use on Covid-19 severity or mortality [7, 14]. However, these studies included few CCB users and were probably lacking power. Recently, an in vitro study on an emerging porcine coronavirus showed that CCB could prevent infection and intracellular calcium homeostasis dysregulation [15]. Regarding beta-blockers, one could hypothesize that they can counteract deleterious sympathetic activation during the SARS-CoV-2induced cytokine storm and severe disease. Further clinical data are needed to explore these early findings.

Our study has some limitations. First, in this retrospective observational design on hospitalized Covid-19 patients, hypertension or antihypertensive exposure could have affected the chance of hospitalization, which may limit the generalizability of our results. Second, although analyses were adjusted on major comorbidities, some unmeasured or unknown confounders may have not been ruled out, including indication bias. We also cannot exclude the role of the underlying disease in the observed differences. Finally, ascertainment of medication uses from EHRs may not capture all drug prescriptions and not reflect actual drug exposure. However, considering antihypertensive agents are chronic treatments, we consider exposure to these drugs up to 6 months prior to hospitalization for Covid-19.

\section{Conclusion}

In summary, in hospitalized patients with hypertension, the chronic use of CCB, beta-blockers, or RAAS inhibitors did not worsen Covid-19. In this study, we did not find any association between the use of RAAS inhibitors and the risk of inhospital death in Covid-19 patients with hypertension. Interestingly, we found a reduced mortality among $\mathrm{CCB}$ and beta-blockers users, which remain significant when considering only CCB or beta-blocker monotherapies. Further studies are needed to confirm this protective role. Our findings, such as previous reports, support the continuation of antihypertensive agents in Covid-19 patients, in line with the current guidelines.

Supplementary Information The online version contains supplementary material available at https://doi.org/10.1007/s10557-021-07155-5.

Acknowledgements The authors thank the EDS AP-HP Covid consortium for integrating the AP-HP Health Data Warehouse team as well as all the AP-HP staff and volunteers who contributed to the implementation of the EDS-Covid database and operating solutions for this database.

Collaborators: AP-HP/Universities/INSERM COVID-19 Research Collaboration; AP-HP COVID CDR Initiative Collaborators: PierreYves Ancel, Alain Bauchet, Nathanaël Beeker, Vincent Benoit, Mélodie Bernaux, Ali Bellamine, Romain Bey, Aurélie Bourmaud, Stéphane Breant, Anita Burgun, Fabrice Carrat, Charlotte Caucheteux, Julien Champ, Sylvie Cormont, Christel Daniel, Julien Dubiel, Catherine Ducloas, Loic Esteve, Marie Frank, Nicolas Garcelon, Alexandre Gramfort, Nicolas Griffon, Olivier Grisel, Martin Guilbaud, Claire Hassen-Khodja, François Hemery, Martin Hilka, Anne Sophie Jannot, Jerome Lambert, Richard Layese, Judith Leblanc, Léo Lebouter, Guillaume Lemaitre, Damien Leprovost, Ivan Lerner, Kankoe Levi Sallah, Aurélien Maire, Marie-France Mamzer, Patricia Martel, Arthur Mensch, Thomas Moreau, Antoine Neuraz, Nina Orlova, Nicolas Paris, Bastien Rance, Hélène Ravera, Antoine Rozes, Elisa Salamanca, Arnaud Sandrin, Patricia Serre, Xavier Tannier, Jean-Marc Treluyer, Damien Van Gysel, Gaël Varoquaux, Jill Jen Vie, Maxime Wack, Perceval Wajsburt, Demian Wassermann, and Eric Zapletal.

\section{Author Contribution}

Study design: LC and AN

Data management and database build process: NG, BR, NP, and ES

Statistical analysis: NB and AN

Result interpretation: $\mathrm{LC}$ and AN

Draft the manuscript: LC

Critical review of the manuscript: $\mathrm{LC}, \mathrm{AN}, \mathrm{EP}, \mathrm{AB}$, and JMT

Approved the manuscript: LC, NB, NG, BR, NP, ES, EP, AB, JMT, and AN

Funding This work was supported by State funding from The French National Research Agency (ANR) under "Investissements d'Avenir" programs (Reference: ANR-10-IAHU-01) and ANR PractikPharma grant (ANR-15-CE23-0028).

Data Availability The data underlying this article will be shared on reasonable request to the corresponding author.

Code Availability The custom code underlying this article will be shared on reasonable request to the corresponding author.

\section{Declarations}

Ethics Approval The study has been performed in accordance with the Declaration of Helsinki and has been approved by the Institutional Review Board of the AP-HP Health Data Warehouse on April 7, 2020 (CSE-20-18_COVID19).

Consent to Participate According to the French law, patients were informed that their data could be used for research.

Consent for Publication Not applicable

Competing Interests The authors declare no competing interests.

\section{References}

1. Gao C, Cai Y, Zhang K, Zhou L, Zhang Y, Zhang X, et al. Association of hypertension and antihypertensive treatment with 
COVID-19 mortality: a retrospective observational study. Eur Heart J. 2020;41:2058-66.

2. Guan W-J, Ni Z-Y, Hu Y, Liang W-H, Ou C-Q, He J-X, et al. Clinical characteristics of coronavirus disease 2019 in China. N Engl J Med. 2020;382:1708-20.

3. Brojakowska A, Narula J, Shimony R, Bander J. Clinical implications of SARS-CoV-2 interaction with renin angiotensin system: JACC Review Topic of the Week. J Am Coll Cardiol. 2020;75: 3085-95.

4. Gurwitz D. Angiotensin receptor blockers as tentative SARS-CoV2 therapeutics. Drug Dev Res. 2020;81:537-40.

5. American Heart Association (AHA), Heart Failure Society of America, Heart Failure Society of America (HFSA), American College of Cardiology (ACC). Patients taking ACE-i and ARBs who contract COVID-19 should continue treatment, unless otherwise advised by their physician [Internet]. Am. Heart Assoc. [cited $2020 \mathrm{Jul}$ 20]. Available from: https://newsroom.heart.org/news/ patients-taking-ace-i-and-arbs-who-contract-covid-19-shouldcontinue-treatment-unless-otherwise-advised-by-their-physician.

6. de Abajo FJ, Rodríguez-Martín S, Lerma V, Mejía-Abril G, Aguilar $\mathrm{M}$, García-Luque A, et al. Use of renin-angiotensin-aldosterone system inhibitors and risk of COVID-19 requiring admission to hospital: a case-population study. Lancet Lond Engl. 2020;395: $1705-14$.

7. Li J, Wang X, Chen J, Zhang H, Deng A. Association of reninangiotensin system inhibitors with severity or risk of death in patients with hypertension hospitalized for coronavirus disease 2019 (COVID-19) infection in Wuhan, China. JAMA Cardiol. 2020;5:16.

8. Neuraz A, Lerner I, Digan W, Paris N, Tsopra R, Rogier A, et al. Natural language processing for rapid response to emergent diseases: case study of calcium channel blockers and hypertension in the COVID-19 pandemic. J Med Internet Res. 2020;22:e20773.

9. Chen T, Wu D, Chen H, Yan W, Yang D, Chen G, et al. Clinical characteristics of 113 deceased patients with coronavirus disease 2019: retrospective study. BMJ. 2020;368:m1091.

10. Zhou F, Yu T, Du R, Fan G, Liu Y, Liu Z, et al. Clinical course and risk factors for mortality of adult inpatients with COVID-19 in Wuhan, China: a retrospective cohort study. Lancet Lond Engl. 2020;395:1054-62.

11. Vaduganathan M, Vardeny O, Michel T, McMurray JJV, Pfeffer MA, Solomon SD. Renin-angiotensin-aldosterone system inhibitors in patients with Covid-19. N Engl J Med. Mass Med Soc. 2020;382:1653-9.

12. Reynolds HR, Adhikari S, Pulgarin C, Troxel AB, Iturrate E, Johnson SB, et al. Renin-angiotensin-aldosterone system inhibitors and risk of Covid-19. N Engl J Med. 2020;382:2441-8.

13. Flacco ME, Acuti Martellucci C, Bravi F, Parruti G, Cappadona R, Mascitelli A, et al. Treatment with ACE inhibitors or ARBs and risk of severe/lethal COVID-19: a meta-analysis. Heart. 2020;106(19): 1519-24. https://doi.org/10.1136/heartjnl-2020-317336.

14. Fosbøl EL, Butt JH, Østergaard L, Andersson C, Selmer C, Kragholm K, et al. Association of angiotensin-converting enzyme inhibitor or angiotensin receptor blocker use with COVID-19 diagnosis and mortality. JAMA. 2020;324:168-77.

15. Bai D, Fang L, Xia S, Ke W, Wang J, Wu X, et al. Porcine deltacoronavirus (PDCoV) modulates calcium influx to favor viral replication. Virology. 2020;539:38-48.

Publisher's Note Springer Nature remains neutral with regard to jurisdictional claims in published maps and institutional affiliations. 